\title{
Search for ultra-high energy photons by complex data of the Yakutsk array
}

\author{
Stanislav Knurenko ${ }^{1, *}$ and Igor Petrov ${ }^{1, * *}$ \\ ${ }^{1}$ Yu.G. Shafer Institute of Cosmophysical Research and Aeronomy SB RAS
}

\begin{abstract}
Complex analysis of the Yakutsk array data in order to find air showers produced by photons with energy $\geq 10^{18} \mathrm{eV}$ is carried out. On the basis of simulations and experimental data, selection criteria are formed and air showers with characteristics close to those criteria are selected. Using the data an upper limit of the integral photon flux in cosmic radiation of ultra-high energies is obtained.
\end{abstract}

\section{Introduction}

Neutral particles due to their physical properties can go a long way in matter before they start to interact with the matter and produce cascades of secondary particles. In the dense atmosphere of the Earth, the neutral particles produce extensive air shower (EAS), and the depth of the maximum development will be near sea level, i.e. these are "young showers". The base of such air showers is the electron-photon component, which scatters to large angles and has long delays relative to particles in the shower core (typically $\tau_{\text {delay }} \leq 5 \mu \mathrm{s}$ ). Hence, in such shower events one needs to expect a large number of peaks from electrons, positrons and photons on the signal scan from scintillation detectors [1-3].

Attention should be made towards inclined air showers with $\theta \geq 60^{\circ}$, if they have a similar picture in the air shower development, namely large number of peaks in the signal scan. This distinguishes air showers produced by primary gamma rays and neutrinos from those produced by primary nuclei with the depth of the maximum height in the atmosphere so, mostly only muons are observed at sea level. In this case, only a single peak would be seen in the signal scan [4].

The aim of our work was to find candidates for EAS produced by primary photons of ultrahigh energy and extra-atmospheric neutrinos. To this end, a comprehensive approach was used, when all components of the shower were analyzed simultaneously: electrons, muons and Cherenkov radiation, including spatio-temporal scans of pulses from scintillation detectors of the Yakutsk EAS apparatus.

\section{Experimental Data}

In order to study characteristics of EAS development from primary gamma rays we modelled the longitudinal development of air showers to sea level using the CORSIKA

\footnotetext{
*e-mail: s.p.knurenko@ikfia.ysn.ru

**e-mail: igor.petrov@ikfia.ysn.ru
}

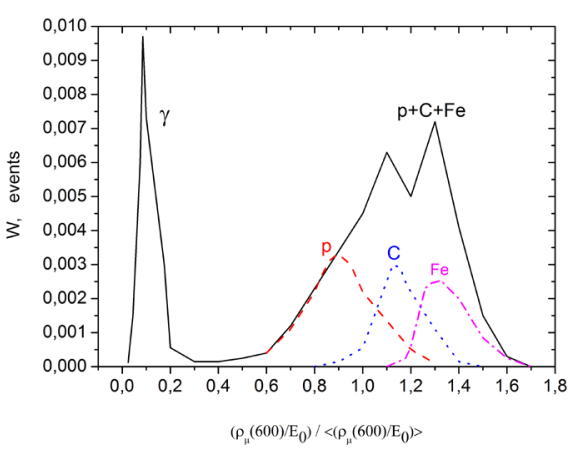

Figure 1. Distribution of showers with energies above $10^{18} \mathrm{eV}$ and zenith angle $\theta \leq 70^{\circ}$. The curves are gamma ray $\gamma$ (solid), proton $\mathrm{p}$ (dash), carbon $\mathrm{C}$ (dot) and iron nucleus Fe (dash-dot) as simulated with QGSjetII-04 [6, 8]

program [5]. Air shower parameters were calculated by QGSjetII-04 [6-8] with thinning, in the case of energies higher than $10^{18} \mathrm{eV}$. In the model we took into account registration condition of each component including the response of each detector and the experimental data analysis.

Experimental data of the electron-photon component of air showers with $\mathrm{E} \geq 1 \mathrm{EeV}$ was obtained by continuous and long-term observation at the Yakutsk array [9]. Radial development of charged particles on the plane of the array was registered by 150 ground scintillation detectors each with area of $2 \mathrm{~m}^{2}$ and 125 underground scintillation detectors with the same size. Longitudinal development was reconstructed by the 50 Cherenkov light detectors [10-13].

In Fig. 1 calculations are shown against the EAS fraction of muons using the density of muon flux at $600 \mathrm{~m}$ from the axis. From Fig. 1 it is seen that the average distribution of each component of cosmic radiation are distributed in certain regions and can be distinguished in the experiment with high enough accuracy of the muon measurements [13]. As can be seen from the model calculations, gamma ray produced air showers have significantly less muons than in proton or iron induced air showers. 


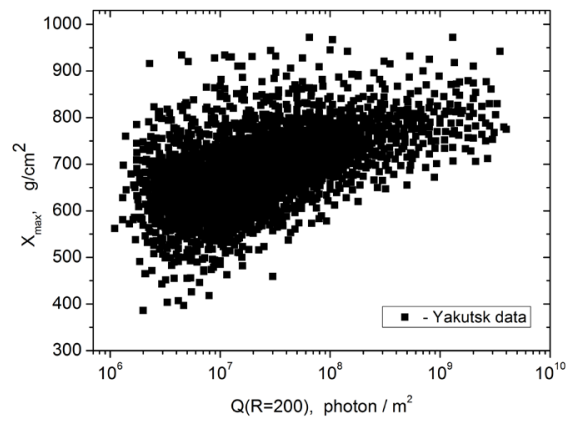

Figure 2. Dependence of $X_{\max }$ from the classification parameter Q (200) - EAS Cherenkov light flux density at a distance of 200 $\mathrm{m}$ from the shower axis. Data obtained in 1973-2014

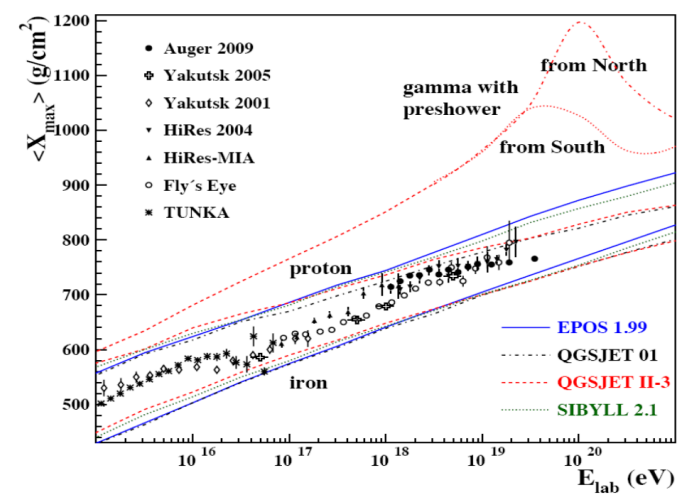

Figure 3. Dependence of $X_{\max }$ on energy. Experimental data comparison with calculations for different hadron interaction models (proton, iron nuclei and high-energy gamma ray). Data obtained in 1973-1993 [17]

Within the accuracy of measurement of $\rho_{\mu}(600)$ at $5-8 \%$ level it is possible to distinguish gamma ray induced air showers.

The longitudinal development of the shower at the Yakutsk array is reconstructed from the detected Cherenkov light data $[14,15]$, using the mathematical apparatus used in solving inverse problems [12, 16].

Using the Cherenkov data database, the distribution of $\mathrm{X}_{\text {max }}$ from the primary energy of the shower was obtained (see Fig. 2 ). It can be seen in Fig. 2 that there are cases of EAS with $\mathrm{X}_{\max } \geq 800 \mathrm{~g} / \mathrm{cm}^{2}$, i.e. have a low maximum development of the shower in relation to the events formed by iron nuclei and even by protons. These showers formed the basis for this analysis.

The averaged data of the Yakutsk array together with the data of other arrays are shown in Fig. 3. Also calculations are shown for some models of hadronic interactions for primary nuclei and high-energy gamma-rays. As can be seen in Fig. 3, air showers from gamma-rays have an $\mathrm{X}_{\text {max }}$ of $100 \mathrm{~g} / \mathrm{cm}^{2}$ lower in the atmosphere than for proton showers with an energy of $10^{19} \mathrm{eV}$. In fact, the cascade curve $\mathrm{X}_{\max }$ for gamma ray showers is near sea level at a depth of $\sim 950 \mathrm{~g} / \mathrm{cm}^{2}$. In this case, there is a narrow cascade mainly consisting of electrons and photons with

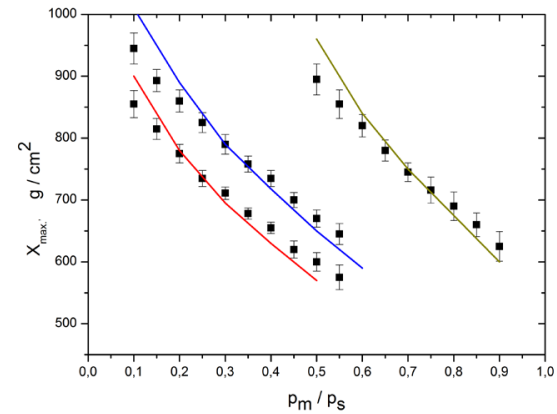

Figure 4. Dependence of the muon fraction on depth of maximum development of electron-photon cascade of air showers at zenith angles $\theta_{1}=18^{\circ}, \theta_{2}=38^{\circ}$ and $\theta_{3}=58^{\circ}$. The fits are made fo

a very low muon content. This distinguishes gamma ray showers from those produced by protons or nuclei of any other element. We can assume that $\mathrm{X}_{\max }$ can be used as the first criterion to search for EAS produced by gamma rays.

Gamma ray produced showers consist of a small number of muons. If the array detects muons based on the number of muons in the shower one can judge the nature of the primary particle i.e. its atomic weight. We can assume this from calculations of muon components by hadron interaction models shown in Fig. 1 [14, 18].

In the Yakutsk array the fraction of muons in the shower is determined by the relation of muon flux density at distances of $600 \mathrm{~m}$ and $1000 \mathrm{~m}$ to the total charged component $\rho_{\mu} / \rho_{\mu+e}$, since these parameters are measured with better accuracy than the total number of muons, $\mathrm{N}_{\mu}$, and charged particles, $\mathrm{N}_{\mu+e}$, in showers with total energy $\mathrm{E}_{0} \geq 10^{18} \mathrm{eV}$. This will be the second criterion to search for showers produced by neutral particles that includes gamma rays and neutrinos.

It is well known that the muon number depends on the height of maximum development of air showers in the atmosphere. That is why the fraction of muons is very sensitive to $\mathrm{X}_{\max }$ of the cascade curve. This follows from calculations based on the QGSjetII-04 model. And the fraction of muons from the proton and iron nucleus, as follows from Fig. 4, is localized in different places, which makes it possible to divide showers into protons and showers from the iron nucleus in this parameter. That feature was used in the present paper to search for neutral particles in cosmic rays.

For this, we used the experimental dependence of $\mathrm{X}_{\max }$ on $\rho_{\mu}(600) / \rho_{s}(600)$, shown in Fig. 4. The fits are calculations made using QGSjetII-04 for different zenith angles. The agreement of calculations with experiment indicates that the QGSjetII-04 model, after its modernization, is close to the description of the experiment on the muon component and the problem of muon deficit is probably solved.

Air showers produced by different primary particles have a maximum development at different depths in the 
atmosphere. Because of this, part of secondary particles (mostly electrons) lose energy by ionization in the air and are eliminated from the cascade process. Then at sea level certain types of particle will arrive: electrons, photons, muons in the case of inclined showers and only muons for strongly inclined showers, which can be seen in the signal time sweep of scintillation detectors. For primary gamma rays and neutrinos the depth of maximum is going to be near the observation level and we can expect scintillation detector response inherent in the electron-photon component of the shower. This is the third criterion by which we can select showers produced by neutral particles.

\section{Estimation of the gamma-ray flux upper limit}

To search for EAS produced by ultra-high energy photons we used the following criteria, which the shower should satisfy to be considered as a shower produced by a neutral particle:

- The depth of the maximum, $\mathbf{X}_{\max }$, of the air shower must be within $850-950 \mathrm{~g} / \mathrm{cm}^{2}$ range;

- The fraction of muons must be $\leq 0.1$ (Fig. 1) [18-22];

- There must be multi-peak structure on the scan of the signal of scintillation detector, specific for "young" showers [4];

The aperture of the Yakutsk array for ultra-high energy showers for the selected time period was equal to:

$$
S_{\text {geom }} \cdot T \cdot \Omega=949.54 \mathrm{~km}^{2} \cdot \mathrm{sr} \cdot \mathrm{g}
$$

The effective exposure after implementation of the coefficients is shown in Table 1.

Table 1. Effective exposure

\begin{tabular}{|c|c|c|c|}
\hline $\mathrm{E}_{0}, \mathrm{eV}$ & $\mathrm{K}_{1}, \%$ & $\mathrm{~K}_{2}, \%$ & $\mathrm{~S}_{e f f . \gamma} \cdot T \cdot \Omega$ \\
\hline $1 \cdot 10^{18}$ & 6.6 & 6.6 & 153.9 \\
\hline $3 \cdot 10^{18}$ & 7.5 & 7.5 & 210.6 \\
\hline $6 \cdot 10^{18}$ & 12.0 & 12.0 & 555.1 \\
\hline $9 \cdot 10^{18}$ & 15.2 & 15.2 & 853.5 \\
\hline
\end{tabular}

The implementation of the coefficients made it possible to take into account the dependence of $S_{\text {eff.y }}$ on the zenith angle and the EAS trigger with the shower selection threshold equal to 2 particles $/ \mathrm{m}^{2}$. In this case, the probability of detecting showers over charged particles was equal to $\mathrm{W}_{p} \geq 0.95$, and the accuracy of the EAS parameters being determined was not worse than $10 \%$.

An upper limit on a mathematical expectation of the number of photons is determined following [24] for $95 \%$ $\mathrm{CL}$ and the number of candidates, assuming to be produced by primary photons of ultra-high energies is shown in Table 2.

The integral flux upper limit for primary gamma-rays follows from the formula:

$$
F_{\gamma}^{95 C L}=\left(n_{\gamma}\left(E_{\gamma}>E_{0}\right)\right) / S_{e f f . \gamma} \cdot T \cdot \Omega
$$

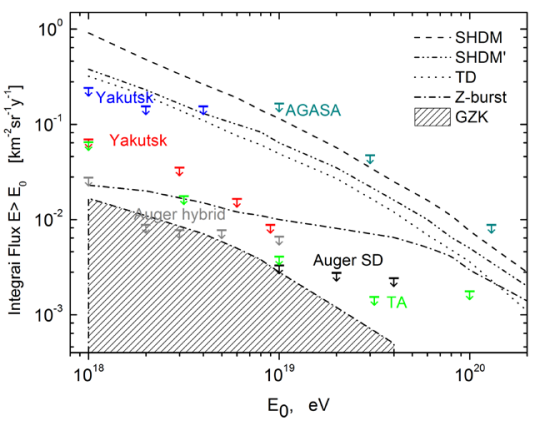

Figure 5. Photon flux limit by the Yakutsk Array data: new [27] and old [28]. Comparison with Auger [29], AGASA [30], TA [31] and the predictions of certain models [24-26, 32]

Table 2. Predicted number of showers produced by primary photons

\begin{tabular}{|c|c|c|c|c|}
\hline Energy, eV & $1 \cdot 10^{18}$ & $3 \cdot 10^{18}$ & $6 \cdot 10^{18}$ & $9 \cdot 10^{18}$ \\
\hline $\mathrm{N}_{\gamma}$ & 4 & 2 & 3 & 2 \\
\hline $\mathrm{S}_{e f f . \gamma}$ & 153.9 & 210.6 & 555.1 & 853.5 \\
\hline $\mathrm{n}_{\gamma}$ & 9.76 & 6.72 & 8.25 & 6.72 \\
\hline $\mathrm{F}^{95 C L_{<}}$ & 0.063 & 0.032 & 0.015 & 0.08 \\
\hline
\end{tabular}

Thus we obtained the integral flux upper limit for photons within 1-10 EeV (Fig. 5)

Fig. 5 shows predictions of certain models for the integral flux of primary gamma-rays, which indicates possible sources [24-26]. Those are super heavy dark matter (SHDM) [24] and SHDM' [26], topological defects (TD) and Z-bursts [24]. The first experimental results obtained at AGASA, Yakutsk (old) and Auger rather tends towards SHDM and SHDM' while newer results obtained lately, including new Yakutsk array results on gamma-ray sources, are closer to GZK mechanism [24].

The difference between early results and new ones is associated with an increase in exposure and models that were used to estimate the flux of photons in cosmic rays from the experimental data of EAS. The refinement of the increased number of muons in the new models caused a change in the selection criteria for showers and, consequently, a decrease in the integral flux of the primary gamma ray.

Hence, based on the results of the work, we obtained empirical estimations of the integral flux upper limit of photons in cosmic rays with energies $\mathrm{E} \geq 1 \mathrm{EeV}$.

\section{Conclusion}

Multicomponent analysis of EAS using the above criteria did not reveal explicit showers produced by a gamma-ray or neutrino [33]. At the same time, calculations based on the QGSJETII model [34] for primary protons, iron nucleus and gamma-rays lead to the conclusion that if we take into account the fluctuations in the muon measurements within the $1 \sigma$ range, the probability of recording 
the EAS from there are neutral particles. Candidates for such showers can be considered as the "muonless" showers found in the Yakutsk EAS array [35]. The presented results (Fig. 5) on the upper limit of the gamma-ray flux can be used to test various astrophysical models (including the search for dark matter) and to establish the nature of the formation of particles such as astro-neutrinos in cosmological space.

In order to study the nature of neutral particles in the region of ultrahigh energies, it is necessary to improve the method of measurements, primarily, for strongly inclined air showers. It is even possible to create specialized arrays, for example, muon telescopes with a large receiving area for registering the muon fraction with an accuracy of 3-5\% in individual EAS events.

\section{Acknowledgment}

The reported study was funded by RFBR according to the research project 16-29-13019.

\section{References}

[1] S. Knurenko, Z. Petrov and Yu. Yegorov, J. Phys. Conf. Ser. 409012090 (2013)

[2] S.P. Knurenko and A. Sabourov, in Proc. of the 33rd ICRC2013, Rio de Janeiro, 2013 [0055]

[3] G.I. Rubtsov, M. Fukushima, D. Ivanov, B.T. Stokes, G.B. Thomson and S.V. Troitsky, EPJ WEb Conf. 53, 05001 (2013)

[4] S. Knurenko, Z. Petrov, Yu. Yegorov and N. Dyachkovsky, Proc 21st ECRS, Kosice (Slovakia), 465 (2008) (2003)

[5] N. Capdevielle, G. Schatz and T. Thouw, Forschungszentrum Karlstruhe Thechnical Report 6019, 90 (1998)

[6] S.P. Knurenko and A.V. Sabourov, Nucl Phys. B, 196, 319 (2009)

[7] T. Pierog and K. Werner, Phys. Rev. Lett. 101, 171101 (2008)

[8] S.S. Ostapchenko, Nucl. Phys. B, 151, 143 (2006)

[9] V.P. Artamonov, B.N. Afanasyev, A.V. Glushkov, A.A. Mikhailov, S.P. Knurenko, Bull. of RAoS. Physics 58, 92 (1994)

[10] M.N. Dyakonov, S.P. Knurenko, V.A. Kolosov et al., Moscow: Science, 339 (1990)

[11] M.N. Dyakonov, S.P. Knurenko, V.A. Kolosov et al., NIM A 248, 244 (1986)

[12] S. Knurenko, V. Kolosov, Z. Petrov et al., Proc. 27th ICRC, Hamburg, 1, 177 (2001)

[13] V.B. Atraskevich, N.N. Kalmykov, G.B. Christiansen, JETP Lett. 33, 236 (1981)

[14] G.K. Garipov, V.M. Grigoryev, N.N. Efremov et al. Proc. 27th ICRC, Hamburg 1, 885-887 (2001)
[15] S.P. Knurenko, V.A. Kolosov, Z.E. Petrov et al., Proc. 27th ICRC, Hamburg 1, 559-561 (2017)

[16] A. Tikhonov, V. Arsenin, Solution of Ill-posed Problems (Winston, NY, 1977) 258

[17] M.N. Dyakonov, A.A. Ivanov, S.P. Knurenko, Proc. 23rd ICRC, Calgary 4, 303 (1993)

[18] S.P. Knurenko, A.A. Ivanov, M.I. Pravdin, A.V. Sabourov and I.Ye. Sleptsov, Nucl. Phys. B. (Proc. Suppl.) 175-176, 201 (2008)

[19] S.P. Knurenko, V.P. Egorova, A.A. Ivanov, V.A. Kolosov, I.T. Makarov, Z.E. Petrov, I.Ye. Sleptsov and G.G. Struchkov, Nucl. Phys. B. 151, 92 (2006)

[20] S.P. Knurenko and A.V. Sabourov, Nucl. Phys. B 196, 319 (2009)

[21] K. Shinozaki, M. Chikawa, M. Fukushima, N. Hayashida, K. Honda, N. Inoue, K. Kadota, F. Kakimoto, K. Kamata, S. Kawaguchi, S. Kawakami, Y. Kawasaki, N. Kawasumi, K. Mase, and S. Mizobuchi, Proc. 28th ICRC. Tsukuba (Japan) 3, 401 (2003).

[22] S.P. Knurenko, A.A. Ivanov, I.Ye. Sleptsov, Bull. of RAoS. Phys. Ser. 69, 363 (2005)

[23] G.J. Feldman and R.D. Cousins, Phys. Rev. D 57, 3873 (1998)

[24] P. Bhattacharjee and G. Sigl, Phys. Rev. 327, 109 (2000) [V. S. Berezinsky and G.T. Zatsepin, Phys. Lett. B 28(6), 423 (1969)].

[25] F. Halzen and D. Hooper, Rep. Prog. Phys. 65, 1025 (2002)

[26] J. Ellis, V.E. Mayes and D.V. Nanopoulos, Phys. Rev. D 74, 115003 (2006)

[27] S.P. Knurenko, I.S. Petrov, JETP Lett. 104, 297 (2016)

[28] A.V. Glushkov, I.T. Makarov, M. I. Pravdin, and I.E. Sleptsov, Phys. Rev. D 82, 041101 (2002).

[29] M.N. Dyakonov, A.A. Ivanov, S.P. Knurenko, V.A. Kolosov and D.D. Krasilnikov, Proc. 17th ICRC 6, 76 (1981)

[30] K. Shinozaki, M. Chikawa, M. Fukushima, N. Hayashida, N. Inoue, K. Honda, K. Kadota, F. Kakimoto, K. Kamata, S. Kawaguchi, S. Kawakami, Y. Kawasaki, N. Kawasumi, A.M. Mahrous and K. Mase, Astrophys. J. 571, L117 (2002)

[31] G.I. Rubtsov (for the Telescope Array Collaboration), Proceedings of Science, 551 (2017).

[32] J.K. Becker, Phys. Rep. 458, 173 (2008).

[33] S.P. Knurenko, A.K. Makarov, M.I. Pravdin, A.V. Sabourov, Bull. Russ. Acad. Sci. Phys. 75, 291 (2011)

[34] S. Ostapchenko, Phys. Lett.B 636, 40 (2006)

[35] A.V. Glushkov, I.T. Makarov, M.I. Pravdin, I.Ye. Sleptsov, D.S. Gorbunov, G.I. Rubtsov and S.V. Troitsky, Phys. Rev. D 82, 041101(R) (2010) 